\title{
Emergence of anisotropic Gilbert damping in ultrathin Fe layers on GaAs(001)
}

\author{
L. Chen ${ }^{1}$, S. Mankovsky², S. Wimmer², M. A. W. Schoen', H. S. Körner', M. Kronseder', D. Schuh', \\ D. Bougeard', H. Ebert ${ }^{2}$, D. Weiss ${ }^{1}$ and C. H. Back (iD) ${ }^{1 \star}$
}

\begin{abstract}
As a fundamental parameter in magnetism, the phenomenological Gilbert damping constant $\alpha$ determines the performance of many spintronic devices. For most magnetic materials, $\alpha$ is treated as an isotropic parameter entering the Landau-LifshitzGilbert equation. However, could the Gilbert damping be anisotropic? Although several theoretical approaches have suggested that anisotropic $\alpha$ could appear in single-crystalline bulk systems, experimental evidence of its existence is scarce. Here, we report the emergence of anisotropic magnetic damping by exploring a quasi-two-dimensional single-crystalline ferromagnetic metal/semiconductor interface-that is, a Fe/GaAs(001) heterojunction. The observed anisotropic damping shows twofold $C_{2 v}$ symmetry, which is expected from the interplay of interfacial Rashba and Dresselhaus spin-orbit interaction, and is manifested by the anisotropic density of states at the Fe/GaAs (001) interface. This discovery of anisotropic damping will enrich the understanding of magnetization relaxation mechanisms and can provide a route towards the search for anisotropic damping at other ferromagnetic metal/semiconductor interfaces.
\end{abstract}

\begin{abstract}
$\mathrm{n}$ the past decades, the damping constant $\alpha$ has been successfully described theoretically_in some cases even quantitatively-using various approaches such as the breathing Fermi-surface model ${ }^{1,2}$, the torque correlation model $^{3}$, scattering theory ${ }^{4,5}$ and the torquetorque correlation within a linear response model ${ }^{6,7}$. On the basis of these works, $\alpha$ is expected to scale as $\alpha \sim n\left(E_{\mathrm{F}}\right) \xi^{2} \tau^{-1}$ under certain circumstances, where $n\left(E_{\mathrm{F}}\right)$ is the density of states at the Fermi level $E_{\mathrm{F}} \xi$ is the strength of the spin-orbit interaction and $\tau$ is the electron momentum scattering time ${ }^{8,9}$. Indeed, the dependences on $n\left(E_{\mathrm{F}}\right)$ (refs ${ }^{9-11}$ ), $\xi$ (refs ${ }^{12,13}$ ) and $\tau$ (ref. ${ }^{14}$ ) have been confirmed separately in a large variety of materials. In general, it is assumed that damping is isotropic. However, several theoretical works ${ }^{15-18}$ have suggested that damping should be anisotropic in single-crystalline ferromagnetic metals, such as bulk Fe, Co and Ni. This prediction is based on the anisotropic electronic structure where the shape of the Fermi surface depends on the orientation of the magnetization direction due to the spin-orbit interaction. The anisotropic electronic structure and thus the anisotropic damping, however, can be dramatically reduced due to smearing of the energy bands in the presence of electron scattering, which makes the experimental observation of the anisotropic damping in bulk materials difficult. So far, only a few experiments ${ }^{19-22}$ have tried to prove the existence of anisotropic damping in bulk magnets but convincing experimental evidence is still lacking.
\end{abstract}

Here, we report the observation of anisotropic Gilbert damping in a quasi-two-dimensional Fe/GaAs(001) system. The idea behind this is to explore the interfacial spin-orbit interaction of a singlecrystalline ferromagnetic metal/semiconductor interface. Our findings differ distinctly from the theoretical predictions made for bulk magnets. The $\mathrm{Fe} / \mathrm{GaAs}$ heterostructure was intensively studied in the past two decades for semiconductor spintronics, and has been utilized, for example, to realize spin injection at room temperature $^{23}$. Recently, interest in this system has been revived in view of spin-orbit electronics, because of the existence of robust spin-orbit fields at the $\mathrm{Fe} / \mathrm{GaAs}$ interface, which can cause a mutual conversion between spin and charge currents at room temperature ${ }^{24}$. The spinorbit fields, including both Bychkov-Rashba- and Dresselhaus-like terms, result from the $C_{2 v}$ symmetry of the interface ${ }^{25}$. Specifically, at the $\mathrm{Fe} / \mathrm{GaAs}(001)$ interface, Fe Bloch states near $E_{\mathrm{F}}$ penetrate into GaAs. Therefore, electrons of Fe 'feel' both Bychkov-Rashba and Dresselhaus spin-orbit interaction at the interface, causing a rich variety of interfacial spin-orbit-related phenomena. It has been found, for example, that the symmetry of anisotropic magnetoresistance ${ }^{26}$ and the polar magneto-optic Kerr effect ${ }^{27}$ of Fe is governed by the twofold interfacial $C_{2 v}$ symmetry rather than its bulk fourfold $C_{4 v}$ symmetry when the thickness of Fe is decreased to a few monolayers (MLs). Here, we address the importance of symmetrybreaking on magnetic damping.

\section{Spin-orbit ferromagnetic resonance}

We use spin-orbit ferromagnetic resonance ${ }^{24,28}$ (SO-FMR) to measure damping. Although the conventional full-film FMR technique also supports the experimental findings, SO-FMR facilitates the detection of magnetization dynamics of ultrathin films by higher sensitivity, and largely avoids extrinsic effects contributing to the FMR linewidth (see Supplementary Information for a comparison between full-film FMR and SO-FMR). Two series of samples, S1 and S2, with different nominal Fe thickness $t$ have been prepared (see Methods). We mainly report measurements from the S1 series (samples from series S2 show similar results). Devices with dimensions of $6.4 \mu \mathrm{m} \times 100.0 \mu \mathrm{m}$ oriented along the [100] direction are defined by employing electron-beam lithography and ion-beam etching (Fig. 1a and see Methods). Fig. 1c shows the typical d.c. voltage spectra of sample $S 1(1.3 \mathrm{~nm})$ for a fixed magnetic-field angle $\varphi_{\mathrm{H}}=125^{\circ}$ at several selected frequencies. Each spectrum can be well fitted by combining a symmetric $\left(L_{\text {sym }}=\Delta H^{2} /\left[4\left(H-H_{\mathrm{R}}\right)^{2}+\right.\right.$ $\left.\left.\Delta H^{2}\right]\right)$ and an antisymmetric Lorentzian $\left(L_{\mathrm{a}-\mathrm{sym}}=-4 \Delta H\left(H-H_{\mathrm{R}}\right) /\right.$ $\left.\left[4\left(H-H_{\mathrm{R}}\right)^{2}+\Delta H^{2}\right]\right), \quad V-V_{\text {offset }}=V_{\text {sym }} L_{\text {sym }}+V_{\text {a-sym }} L_{\text {a-sym }}$, where $H$ is the external magnetic field, $H_{\mathrm{R}}$ is $H$ at FMR, $\Delta H$ is the full-width at half-maximum, $V_{\text {sym }}\left(V_{\text {a-sym }}\right)$ is the magnitude of the symmetric

'Institute of Experimental and Applied Physics, University of Regensburg, Regensburg, Germany. ${ }^{2}$ Department of Chemistry, University of Munich, Munich, Germany. *e-mail: christian.back@ur.de 


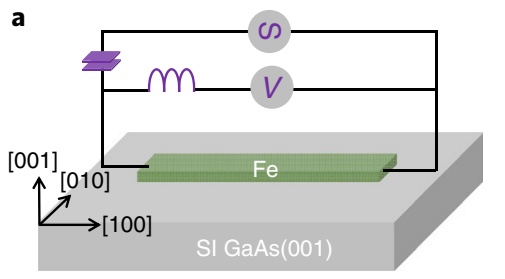

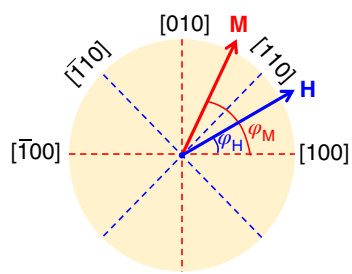

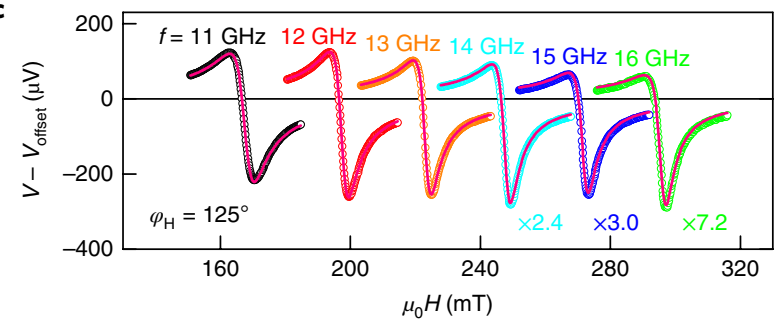

Fig. 1 | Typical d.c. voltage spectra induced by ferromagnetic resonance at the $\mathrm{Fe} / \mathrm{GaAs}(001)$ interface. a, Schematic of the device structure and spin-orbit ferromagnetic resonance set-up. Microwaves pass through a bias-tee and then into the Fe stripe, which is grown on a semi-insulating (SI) $\mathrm{GaAs}(001)$ substrate. The stripe is $100 \mu \mathrm{m}$ long and $6.4 \mu \mathrm{m}$ wide. The injected microwave current $\mathbf{j}(t)$ generates time-dependent spin-orbit fields, which drive the magnetization dynamics $\mathbf{M}(t)$, causing timedependent resistance $R(t)$ via the anisotropic magnetoresistance effect. A rectified d.c. voltage emerges from the coupling of $\mathbf{j}(t)$ and $R(t)$ at ferromagnetic resonance. $\mathbf{b}$, Crystal orientation of $\mathrm{GaAs}$ and definition of the angles of magnetization $\varphi_{\mathrm{M}}$ and external magnetic field $\varphi_{\mathrm{H}}$ with respect to the [100] direction $\mathbf{c}$, Typical d.c. voltage spectra for several selected frequencies of $S 1(1.3 \mathrm{~nm})$ measured at $\varphi_{\mathrm{H}}=125^{\circ}$ and room temperature. Each spectrum can be fitted by a combination of symmetric and antisymmetric Lorentzian functions, from which the FMR resonance field $H_{R}$ and the linewidth $\Delta H$ are obtained.

(antisymmetric) component of the d.c. voltage and $V_{\text {offset }}$ is the offset background voltage. The fitting procedure gives values for $V_{\text {sym }}$, $V_{\text {a-sym }}, H_{\mathrm{R}}$ and $\Delta H$. The magnitude of $V_{\text {sym }}$ and $V_{\text {a-sym }}$ is related to the magnitude of the spin-orbit fields ${ }^{24}, H_{\mathrm{R}}$ is connected to the magnetic anisotropy and $\Delta H$ is linked to the magnetic damping, which we will focus on below.

\section{Magnetic-field-angle dependence of linewidth}

Fig. 2a shows the dependence of linewidth $\Delta H$ on the magneticfield angle $\varphi_{\mathrm{H}}$ for sample $S 1(1.9 \mathrm{~nm})$ measured at microwave frequency $f=12 \mathrm{GHz}$. $\Delta H$ strongly depends on $\varphi_{\mathrm{H}}: \Delta H$ exhibits two peaks around the [110] direction, and $\Delta H$ for the [110] and [110] orientation has, within experimental accuracy, the same magnitude as indicated by the horizontal dashed line. The angular variation of $\Delta H$ can be ascribed to the in-plane magnetic anisotropy, typical for thin Fe films grown on GaAs (001). The angular dependence can be fitted by ${ }^{24}$

$$
\mu_{0} \Delta H=\mu_{0} \Delta[\operatorname{Im}(\chi)]+\mu_{0} \Delta H_{0}
$$

where $\mu_{0}$ is the magnetic permeability of free space, $\Delta H_{0}$ is the zero-frequency inhomogeneous linewidth and $\Delta[\operatorname{Im}(\chi)]$ is the linewidth of the imaginary part of the dynamic magnetic susceptibility $\operatorname{Im}(\chi) \operatorname{Im}(\chi)$ is obtained by solving the Landau-LifshitzGilbert equation ${ }^{24}$

$$
\operatorname{Im}(\chi)=\frac{\alpha \sqrt{H_{1}^{\mathrm{R}} H_{2}^{\mathrm{R}}}\left[H_{1} H_{1}+H_{1}^{\mathrm{R}} H_{2}^{\mathrm{R}}\right] M}{\left(H_{1} H_{2}-H_{1}^{\mathrm{R}} H_{2}^{\mathrm{R}}\right)^{2}+\alpha^{2} H_{1}^{\mathrm{R}} H_{2}^{\mathrm{R}}\left(H_{1}+H_{2}\right)^{2}}
$$
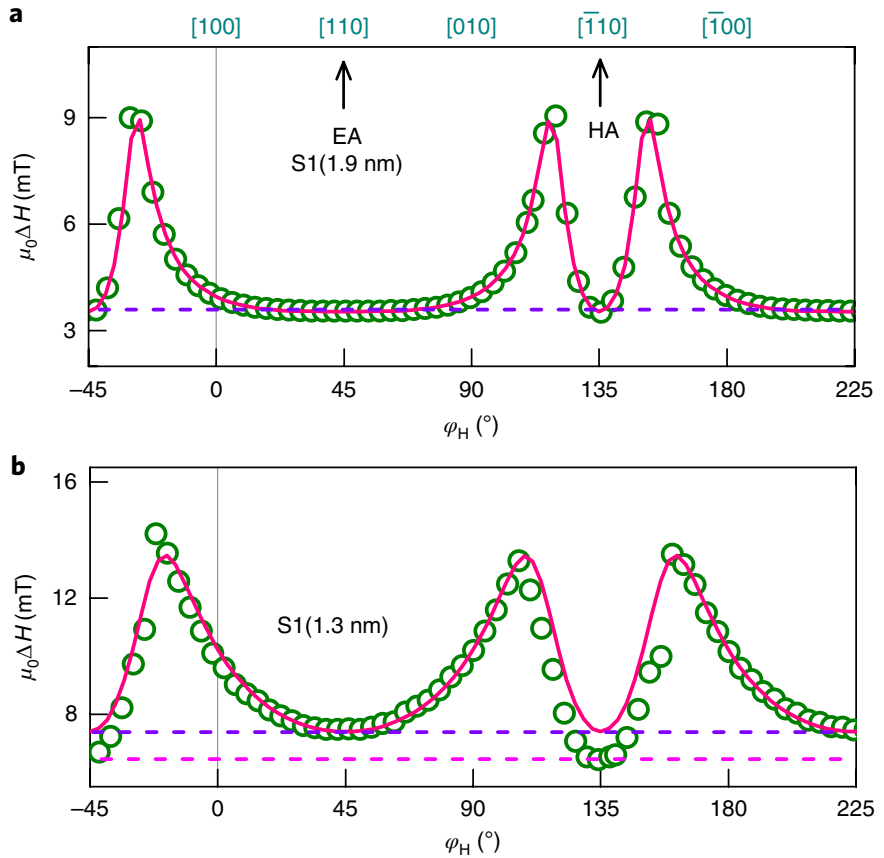

Fig. 2 | Magnetic-field-angle dependence of the linewidth. a,b, Magneticfield-angle $\varphi_{H}$ dependence of the linewidth $\Delta H$ for $\mathrm{S} 1(1.9 \mathrm{~nm})(\mathbf{a})$ and $\mathrm{S} 1(1.3 \mathrm{~nm})(\mathbf{b})$ measured at a microwave frequency of $12 \mathrm{GHz}$ and room temperature. The solid lines in $\mathbf{a}$ and $\mathbf{b}$ are calculated by equation (1) using an isotropic damping constant and $\Delta H_{0}=0 \mathrm{mT}$, which can reasonably reproduce the experimental results for S1 $(1.9 \mathrm{~nm})$. However, for S1 $(1.3 \mathrm{~nm})$, a distinctly smaller linewidth around the [110] orientation appears (see the horizontal dashed lines), and the calculation using a scalar damping constant cannot reproduce the experimental results. These results indicate the emergence of anisotropic damping when reducing the Fe thickness on $\mathrm{GaAs}(001)$. Here, EA represents the magnetic easy axis [110] and $\mathrm{HA}$ represents the magnetic hard axis [ $\overline{1} 10]$.

with $H_{1}=H \cos \left(\varphi_{\mathrm{M}}-\varphi_{\mathrm{H}}\right)+H_{\mathrm{K}}+H_{\mathrm{B}}\left(3+\cos 4 \varphi_{\mathrm{M}}\right) / 4-H_{\mathrm{U}} \sin ^{2}\left(\varphi_{\mathrm{M}}-45^{\circ}\right)$ and $H_{2}=H \cos \left(\varphi_{\mathrm{M}}-\varphi_{\mathrm{H}}\right)+H_{\mathrm{B}} \cos 4 \varphi_{\mathrm{M}}-H_{\mathrm{U}} \sin 2 \varphi_{\mathrm{M}}$. Here $\varphi_{\mathrm{M}}$ is the magnetization angle, $H_{\mathrm{K}}$ is the effective perpendicular magnetic anisotropy field including the demagnetization field, $H_{\mathrm{B}}$ is the biaxial magnetic anisotropy field along $\langle 100\rangle, H_{\mathrm{U}}$ is the in-plane uniaxial magnetic anisotropy field along the [110] orientation and $H_{1}{ }^{\mathrm{R}}\left(H_{2}{ }^{\mathrm{R}}\right)$ is $H_{1}\left(H_{2}\right)$ at $H_{\mathrm{R}}$. By using values of $H_{\mathrm{K}}, H_{\mathrm{B}}$ and $H_{\mathrm{U}}$, obtained by analysing the $\varphi_{\mathrm{H}}$ dependence of $H_{\mathrm{R}}$ (see Supplementary Information), the $\varphi_{\mathrm{H}}$ dependence of $\Delta H$ of $S 1(1.9 \mathrm{~nm})$ can be well reproduced by using equation (1) with an isotropic $\alpha$ of 0.0044 and $\Delta H_{0}=0 \mathrm{mT}$, which is shown by a solid line in Fig. 2a.

Figure $2 \mathrm{~b}$ shows the same kind of data as Fig. 2a, but for sample $\mathrm{S} 1(1.3 \mathrm{~nm})$ with a thinner Fe film. The variation of $\Delta H$ around [110] is stronger than that for $S 1(1.9 \mathrm{~nm})$ due to a larger uniaxial magnetic anisotropy field (see Supplementary Information). The key difference in these two data sets is that the magnitude of $\Delta H$ of $\mathrm{S} 1(1.3 \mathrm{~nm})$ along [110] is significantly larger than that along [110]. If we fit the data the same way as for $\mathrm{S} 1(1.9 \mathrm{~nm}$ ) (that is, by using an isotropic $\alpha$ of 0.0086 and $\Delta H_{0}=0 \mathrm{mT}$ ), the result (solid line in Fig. 2b) deviates distinctly from that of the experiment for the [i110] orientation. The smaller value of $\Delta H$ around the [i10] orientation is a first indication of an anisotropic damping for reduced Fe thickness.

\section{Frequency dependence of linewidth}

Besides the measurements of the angular dependence of the linewidth, analysing the dependence of $\Delta H$ on frequency $f$ is also a well-accepted method to extract the magnitude of $\alpha$ with higher 
accuracy. Fig. 3a shows the frequency dependence of linewidth $\Delta H$ for $\mathrm{S} 1(1.9 \mathrm{~nm})$ along the $[110]$ and $[\overline{1} 10]$ orientations. For both directions, $\Delta H$ is linear in $f$ with the same slope, indicating isotropic damping, which is consistent with the results shown in Fig. 2a. However, the corresponding data for $S 1(1.3 \mathrm{~nm})$, presented in Fig. $3 \mathrm{~b}$, show distinctly different slopes for $\Delta H$ along [110] and [110], indicating the existence of anisotropic damping. Corresponding data taken from sample series S2 are shown in the insets of Fig. 3a,b.

For directions other than [110] and [1 10], $\Delta H(f)$ becomes nonlinear (for example, $\varphi_{\mathrm{H}}=75^{\circ}$, Fig. $3 \mathrm{~d}$ ) or even non-monotonic (for example, $\varphi_{\mathrm{H}}=115^{\circ}$, Fig. 3f) as $\varphi_{\mathrm{H}}$ increases from $45^{\circ}$ to $135^{\circ}$. This nonlinearity can be ascribed to the magnetic dragging effect stemming from the strong in-plane magnetic anisotropy: $\varphi_{\mathrm{M}}$ deviates from $\varphi_{\mathrm{H}}$ for all angles except for the [110] and [1 10] orientations (see Supplementary Information). Both the nonlinear and linear $f$ dependences of $\Delta H$ of $S 1(1.3 \mathrm{~nm})$ can be well reproduced by equation (1), which connects the linewidth to the dynamic magnetic susceptibility $\operatorname{Im}(\chi)$. The best fit to the data shown in Fig. $3 \mathrm{c}-\mathrm{f}$ (blue solid lines) using equation (1) suggests that the magnitude of $\alpha$ of $S 1(1.3 \mathrm{~nm})$ gradually increases from the [110] to the [110] direction. To confirm the isotropic damping in thicker Fe films, we show in the Supplementary Information that the frequency dependence of $\Delta H$ for $\mathrm{S} 1(1.9 \mathrm{~nm})$ along all orientations can be well fitted by an isotropic $\alpha$ of 0.0038 .

Besides the intrinsic damping and $\Delta H_{0}$, extrinsic effects, such as two-magnon scattering ${ }^{29-31}$ and mosaicity broadening ${ }^{31,32}$, could in principle contribute to the observed FMR linewidth. We confirm in the Supplementary Information that the extrinsic contributions are negligibly small, and the main contribution is from intrinsic damping due to the high quality of our samples.

We propose that equation (1) is general in analysing both the magnetic-field angle and the frequency dependence of the FMR linewidth. In particular, it applies to single-crystalline materials with in-plane anisotropy and gives exactly the same results as a formula previously suggested ${ }^{33,34}, \Delta H=\alpha\left(H_{1+} H_{2}\right)\left|\mathrm{d}(\omega / \gamma) / \mathrm{d} H_{\mathrm{R}}\right|^{-1}$, where $\omega(=2 \pi f)$ is the angular frequency and $\gamma$ represents the gyromagnetic ratio. In the absence of in-plane magnetic anisotropy $\left(H_{\mathrm{B}}=H_{\mathrm{U}}=0\right)$, $\Delta H$ has no angular dependence and equation (1) can be simplified to the well-accepted linear form, $\mu_{0} \Delta H=2 \alpha(\omega / \gamma)+\mu_{0} \Delta H_{0}$, which has been widely used for amorphous or polycrystalline materials such as Py.

\section{Angle and thickness dependence of damping}

The reproducible magnitude (see insets of Fig. 3a,b and Supplementary Information for even larger anisotropies appearing in S2) of the damping constant as a function of magnetic-field angles is summarized in Fig. 4a,b. A clear anisotropic behaviour with twofold symmetry is seen for $S 1(1.3 \mathrm{~nm})$, while for $S 1(1.9 \mathrm{~nm})$, damping is isotropic. Note that the difference in thickness is only $0.6 \mathrm{~nm}(\sim 4 \mathrm{ML})$, which shows that the appearance of anisotropic damping is abrupt, indicating an interface-induced origin (a similar behaviour has also been observed for S2). The magnitude of $\alpha$ as function of the inverse thickness $t^{-1}$ is shown in Fig. 4c. Obviously, $\alpha$ changes from isotropic to anisotropic behaviour as the Fe thickness is decreased below a critical thickness. Furthermore, the magnitude of $\alpha$ increases as $t$ decreases. Two mechanisms could be responsible
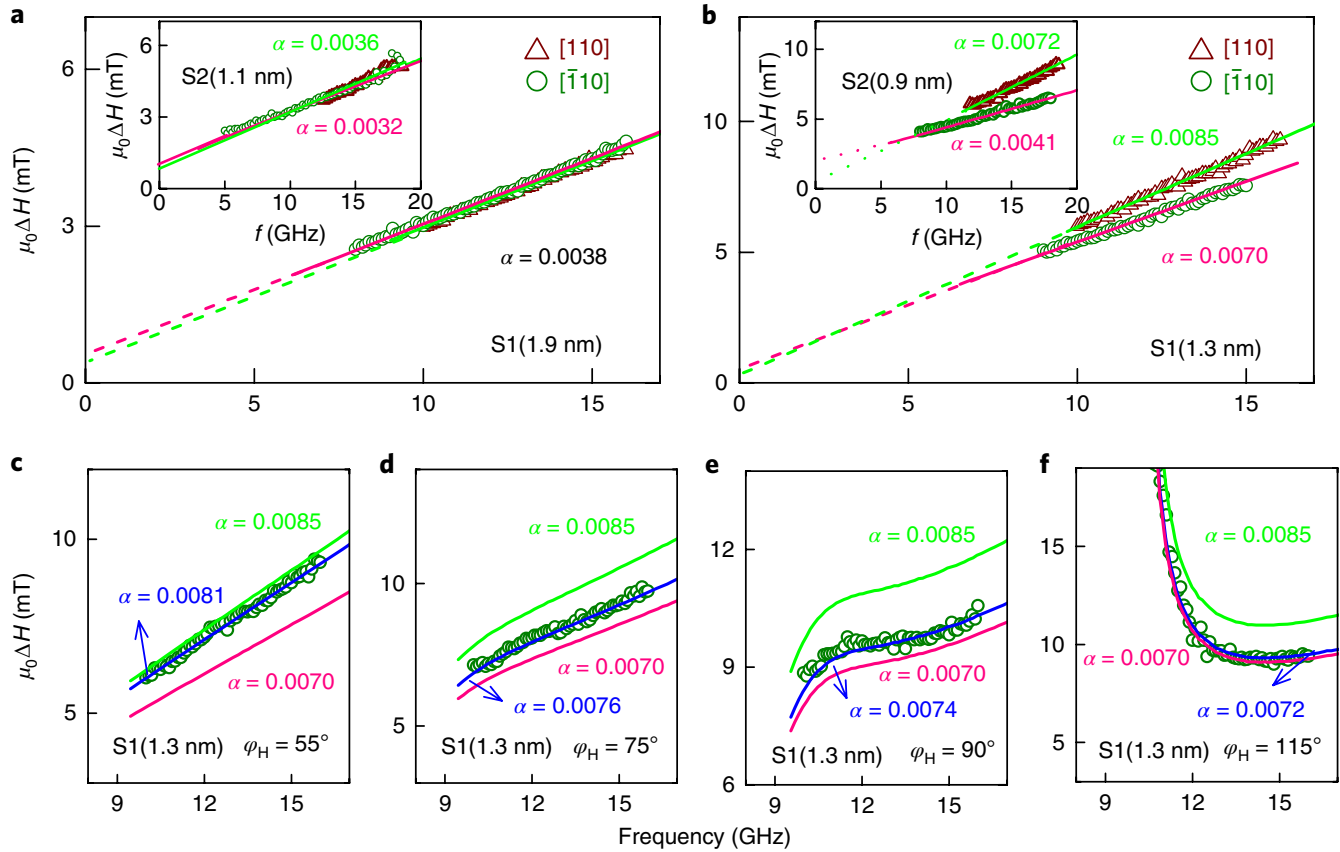

Fig. 3 | Microwave-frequency dependence of linewidth along various crystallographic orientations. a, Dependence of the linewidth $\Delta H$ on microwave frequency $f$ for $\mathrm{S} 1(1.9 \mathrm{~nm})$ with $\mathbf{H}$ along $[110]\left(\varphi_{\mathrm{H}}=45^{\circ}\right)$ and $[\overline{1} 10]\left(\varphi_{\mathrm{H}}=135^{\circ}\right)$. Both directions show the same slope. The green and pink solid lines are calculated by equation (1) using an isotropic damping constant $\alpha$ of 0.0038 . The dashed lines are extrapolations of the calculations, from which the magnitude of $\Delta H_{0}$ is obtained. $\mathbf{b}$, Frequency $f$ dependence of the linewidth $\Delta H$ for $\mathrm{S1}(1.3 \mathrm{~nm})$ for $\mathbf{H}$ along [110] and [110]. The green line is calculated by equation (1) using a damping constant $\alpha$ of 0.0085 , while for the pink line, to fit the [110] data best, $\alpha$ of 0.0070 has been used. These results show that anisotropic damping emerges for reduced Fe thickness. The insets in $\mathbf{a}$ and $\mathbf{b}$ display the same plots for another set of samples, S2(1.1 nm) and S2(0.9 nm), demonstrating that the magnitude of damping anisotropy increases with reduced Fe thickness. Note that the actual thickness may differ from the nominal thickness (see Methods), and quantitative comparison only makes sense for intra-series samples. c-f, $\Delta H(f)$ of $\operatorname{S1}(1.3 \mathrm{~nm})$ for $\varphi_{\mathrm{H}}=55^{\circ}(\mathbf{c}), \varphi_{\mathrm{H}}=75^{\circ}(\mathbf{d})$, $\varphi_{\mathrm{H}}=90^{\circ}(\mathbf{e})$ and $\varphi_{\mathrm{H}}=115^{\circ}(\mathbf{f})$. The green solid lines in $\mathbf{c}-\mathbf{f}$ are calculated using equation (1) with a damping constant $\alpha$ of 0.0085 ; for the pink solid lines, $\alpha=0.0070$ is used and the blue lines are calculated with the corresponding $\alpha$ values indicated. $\mathbf{b}-\mathbf{f}$ show that the magnitude of $\alpha$ gradually increases from [1110] to [110] orientations for S1(1.3nm). 
for the observed enhancement. One is the electronic band effect in the vicinity of the interface, and the other one is spin pumping. In the isotropic regime (above $1.9 \mathrm{~nm}$ ), $\alpha$ scales linearly with $t^{-1}$ and the enhancement of $\alpha$ could be understood in terms of spin pumping ${ }^{35}$, where pure spin currents are excited in the Fe layer and then absorbed at the interfacial Fe layer. This process can cause spin-tocharge conversion at the interface, which is known as the spin galvanic effect and has been proven experimentally ${ }^{24}$. According to the theory of spin pumping in ferromagnetic metal/heavy metal bilayer structures $^{35}, \alpha=\alpha_{0}+g_{\text {eff }} \uparrow \gamma \hbar /(4 \pi M t)$, where $\alpha_{0}$ is the bulk damping of Fe, $g_{\text {eff }} \uparrow \downarrow$ is the effective spin mixing conductance and $\hbar$ is the Planck constant. By fitting $\alpha$ against $t^{-1}$ in the isotropic regime, we obtain $g_{\text {eff }}{ }^{\uparrow}=2.8 \times 10^{18} \mathrm{~m}^{-2}$. This value is comparable to the magnitude in ferromagnetic metal/heavy metal bilayer structures ${ }^{36}$. In the anisotropic regime (below $1.9 \mathrm{~nm}$ ), the magnitude of $\alpha$ shows a dramatic enhancement ( 2 times). Two possibilities could lead to this enhancement: one reason is an enhanced $g_{\text {eff }} \uparrow$ due to the reduction of the back-flowing spin current in the thin-Fe regime, where spin current generation and absorption layers start to merge. The other reason could be an enhanced 'sensing' of the interfacial spin-orbit interaction when Fe approaches the interface, as evidenced from the first-principle calculations (see Supplementary Information).

\section{Discussion}

Finally, we discuss the mechanism of anisotropic damping. Previous results on anisotropic damping measured on Fe/InAs(001) have been related to a growth-induced structural anisotropy ${ }^{19,37}$. However, this is not the case for $\mathrm{Fe} / \mathrm{GaAs}(001)$ since for Fe films thicker than $5 \mathrm{MLs}$, it is found that there is no in-plane distortion and the structure has fourfold symmetry ${ }^{38}$. We also noticed that the twofold symmetry of the anisotropic damping coincides with the symmetry observed for tunnelling anisotropic magnetoresistance ${ }^{39}$, crystalline anisotropic magnetoresistance ${ }^{26}$ and the polar magneto-optic Kerr effect ${ }^{27}$. This follows, as was shown ${ }^{25}$, from the $C_{2 v}$ symmetry of the spin-orbit energy splitting governed by the $C_{2 v}$ symmetry of the Fe/GaAs(001) interface. Since the Gilbert damping and its anisotropy are determined by the electronic properties of the Fe films, monitoring the electronic structure, in particular the effect of interfacial symmetry-breaking on the electronic structure, should provide a deeper understanding of the experimental observations. With this in mind, first-principle calculations of the electronic structure and the Gilbert damping parameter have been performed using the Korringa-Kohn-Rostoker Green function method $^{40}$ (see Supplementary Information). As shown in Fig. 4a, the density of states $n\left(E_{\mathrm{F}}\right)$ of interfacial Fe films is anisotropic, and depends on the orientation of the in-plane magnetization, in full agreement with the observed symmetry of experimentally obtained anisotropic damping. Here, interfacial spin-orbit coupling is made responsible for the anisotropic electronic structure, by performing the calculation in a parameter-free way using the Dirac formalism. This ab initio approach shows that the interfacial spin-orbit interactions modify the electronic structure on varying the magnetization orientation, with the modification stemming mainly from the interfacial Fe $d$ states, as monitored, for example, by the change of the orbital polarization of the Fe $d$ states at the Fe/GaAs (001) interface (see Supplementary Figs. 19 and 20). For Fe layers a

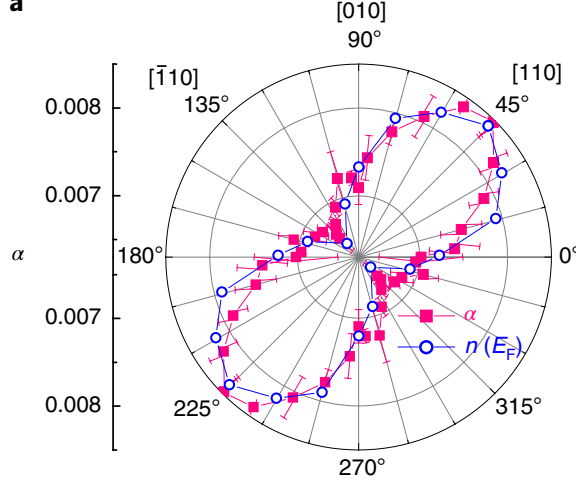

b

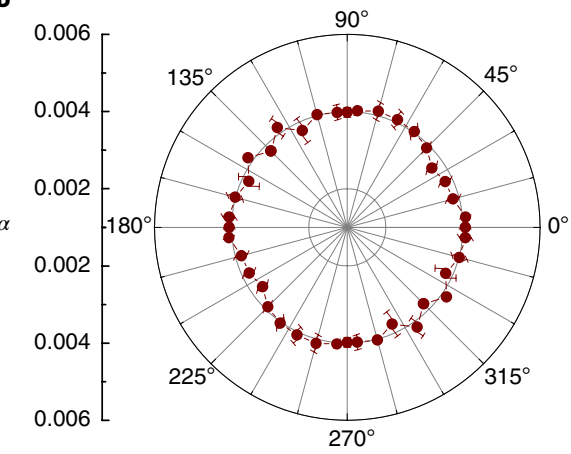

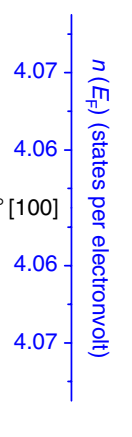

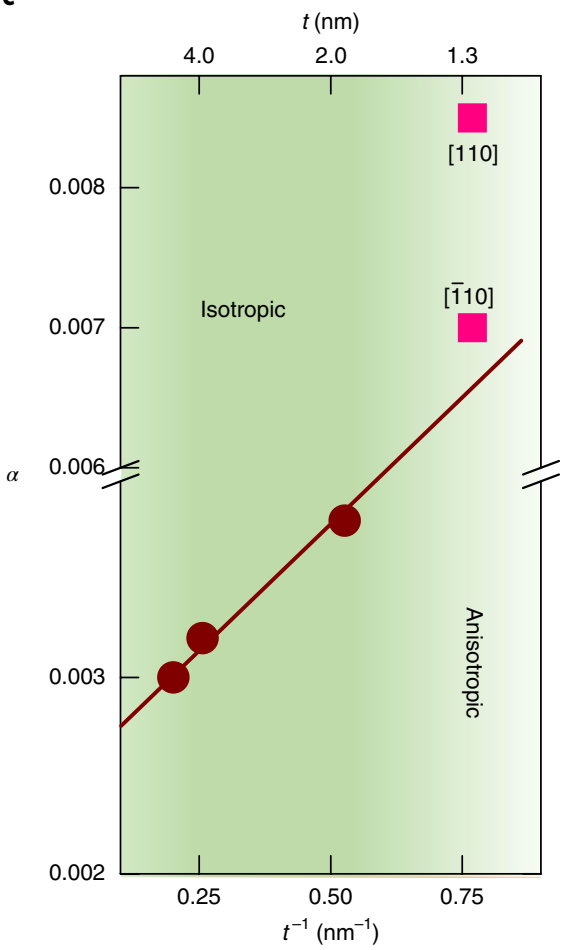

Fig. 4 | Magnetic-field-angle and Fe-thickness dependence of damping. a,b, Magnetic-field-angle $\varphi_{H}$ dependence of the damping constant $\alpha$ for $\mathrm{S} 1(1.3 \mathrm{~nm}$ ) (a) and S1(1.9 nm) (b). Isotropic damping is observed for S1(1.9 nm). However, for S1(1.3 nm), a larger $\alpha$ along $<110>$ is observed, and $\alpha$ gradually decreases until approaching $\langle\overline{1} 10>$. The anisotropic damping shows twofold symmetry, which results from the anisotropic density of states at the Fe/GaAs interface, as shown by open symbols in $\mathbf{a}$. The error bars in $\mathbf{a}$ and $\mathbf{b}$ are the standard deviation obtained from the fit. $\mathbf{c}$, Inverse thickness $t^{-1}$ dependence of the damping constant $\alpha$. The circles represent isotropic damping while squares represent $\alpha$ along [110] and [110] for S1(1.3nm). The solid line is the fit from spin pumping theory, from which the effective mixing conductance $g_{\text {eff }}{ }^{1 \downarrow}$ is determined to be $2.8 \times 10^{18} \mathrm{~m}^{-2}$ and bulk damping $\alpha_{0}=0.0026$. 
away from the interface, the calculation shows that the anisotropy of $n\left(E_{\mathrm{F}}\right)$ quickly disappears (Supplementary Fig. 18), evidencing a reduced impact from the interface. On the basis of these observations, one may expect that, for thicker Fe films, the damping should approach the bulk value. In fact, for $9 \mathrm{ML}$ and $15 \mathrm{ML}$ Fe films, the calculated $\alpha$ values are 0.014 and 0.009 for the [110] orientation, and 0.027 and 0.012 for the [110] orientation, which shows that the magnitude of the anisotropy $\left(\alpha_{[110]}-\alpha_{[-110]}\right) / \alpha_{[-110]}$ decreases from 1.25 for the 9-ML-thick sample to 0.56 for the 15-ML-thick sample (see Supplementary Information). This also explains the experimental finding that the magnitude of the damping diminishes and its anisotropy disappears rapidly when the thickness of the Fe film increases. Moreover, the close relation between the damping parameter $\alpha$ and $n\left(E_{\mathrm{F}}\right)$ suggests that intra-band electron scattering dominates the damping via the breathing Fermi-surface mechanism, which corresponds to the conductivity-like regime.

\section{Methods}

Methods, including statements of data availability and any associated accession codes and references, are available at https://doi. org/10.1038/s41567-018-0053-8.

Received: 25 July 2017; Accepted: 19 January 2018; Published online: 5 March 2018

\section{References}

1. Kamberský, V. On ferromagnetic resonance damping in metals. Czech. J. Phys. B 26, 1366-1383 (1976).

2. Thonig, D. \& Henk, J. Gilbert damping tensor within the breathing Fermi surface model: anisotropy and non-locality. New. J. Phys. 16, 013032 (2014).

3. Gilmore, K., Idzerda, Y. U. \& Stiles, M. D. Identification of the dominant precession-damping mechanism in $\mathrm{Fe}, \mathrm{Co}$, and $\mathrm{Ni}$ by first-principles calculations. Phys. Rev. Lett. 99, 027204 (2007).

4. Brataas, A., Tserkovnyak, Y. \& Bauer, G. E. W. Scattering theory of Gilbert damping. Phys. Rev. Lett. 101, 037207 (2008).

5. Liu, Y., Starikov, A. A., Yuan, Z. \& Kelly, P. J. First-principles calculations of magnetization relaxation in pure $\mathrm{Fe}, \mathrm{Co}$, and $\mathrm{Ni}$ with frozen thermal lattice disorder. Phys. Rev. B 84, 014412 (2011).

6. Mankovsky, S., Ködderitzsch, D., Woltersdorf, G. \& Ebert, H. First-principles calculation of the Gilbert damping parameter via the linear response formalism with application to magnetic transition metals and alloys. Phys. Rev. B 87, 014430 (2013).

7. Turek, I. et al. Nonlocal torque operators in $a b$ initio theory of the Gilbert damping in random ferromagnetic alloys. Phys. Rev. B 92, 214407 (2015).

8. Garate, I. \& MacDonald, A. Gilbert damping in conducting ferromagnets. II. Model tests of the torque-correction formula. Phys. Rev. B 79, 064404 (2009).

9. Mizukami, S. et al. Long-lived ultrafast spin precession in manganese alloys films with a large perpendicular magnetic anisotropy. Phys. Rev. Lett. 106, 117201 (2011).

10. Schoen, M. A. W. et al. Ultra-low magnetic damping of a metallic ferromagnet. Nat. Phys. 12, 839-842 (2016).

11. Oogane, M. et al. Gilbert magnetic damping constant of epitaxially grown Co-based Heusler alloy thin films. Appl. Phys. Lett. 96, 252501 (2010).

12. Oogane, M. et al. Magnetic damping in ferromagnetic thin films. Jpn. J. Appl. Phys. 45, 3889-3891 (2006).

13. He, P. et al. Quadratic scaling of intrinsic Gilbert damping with spin-orbital coupling in $L 1_{0}$ FePdPt films: experiments and ab initio calculations. Phys. Rev. Lett. 110, 077203 (2013).

14. Bhagat, S. M. \& Lubitz, P. Temperature variation of ferromagnetic relaxation in the $3 d$ transition metals. Phys. Rev. B 10, 179-185 (1974).

15. Safonov, V. L. Tensor form of magnetic damping. J. Appl. Phys. 91, 8653-8655 (2002)

16. Steiauf, D. \& Fähnle, M. Damping of spin dynamics in nanostructures: An ab initio study. Phys. Rev. B 72, 064450 (2005).

17. Seib, J., Steiauf, D. \& Fähnle, M. Linewidth of ferromagnetic resonance for systems with anisotropic damping. Phys. Rev. B 79, 092418 (2009).

18. Gilmore, K. \& Stiles, M. D. Anisotropic damping of the magnetization dynamics in Ni, Co, and Fe. Phys. Rev. B 81, 174414 (2010).

19. Meckenstock, R. et al. Anisotropic Gilbert damping in epitaxial Fe films on InAs (001). J. Mag. Mag. Mater. 272-276, 1203-1204 (2004).
20. Zhai, Y. et al. A study on ferromagnetic resonance linewidth of single crystalline ultrathin Fe film grown on GaAs substrate. J. Appl. Phys. 101, 09D120 (2007).

21. Yilgin, R. et al. Anisotropic intrinsic damping constant of epitaxial $\mathrm{Co}_{2} \mathrm{MnSi}$ Heusler alloy films. Jpn. J. Appl. Phys. 46, L205-L208 2007).

22. Kasatani, Y. \& Nozaki, Y. Crystallographic anisotropy of the intrinsic Gilbert damping for single-crystalline Fe film. J. Magn. Soc. Jpn. 39, 221-226 (2015)

23. Zhu, H. J. et al. Room-temperature spin injection from Fe to GaAs. Phys. Rev. Lett. 87, 016601 (2001).

24. Chen, L. et al. Robust spin-orbit torque and spin-galvanic effect at the Fe/GaAs (001) interface at room temperature. Nat. Commun. 7, 13802 (2016).

25. Gmitra, M. et al. Magnetic control of spin-orbit fields: A first-principles study of Fe/GaAs junctions. Phys. Rev. Lett. 111, 036603 (2013).

26. Hupfauer, T. et al. Emergence of spin-orbit fields in magnetotransport of quasi-two-dimensional iron on gallium arsenide. Nat. Commun. 6, 7374 (2015).

27. Buchner, M. et al. Anisotropic polar magneto-optic Kerr effect of ultrathin Fe/GaAs (001) layers due to interfacial spin-orbit interaction. Phys. Rev. Lett. 117, 157202 (2016).

28. Fang, D. et al. Spin-orbit-driven ferromagnetic resonance. Nat. Nanotech. 6, 413-417 (2011).

29. Arials, R. \& Mills, D. L. Extrinsic contributions to the ferromagnetic resonance response of ultrathin film. Phys., Rev. B 60, 7395-7409 (1999).

30. McMichael, R. D. et al. Localized ferromagnetic resonance in inhomogeneous thin films. Phys. Rev. Lett. 90, 227601 (2003).

31. Zakeri, Kh. et al. Spin dynamics in ferromagnets: Gilbert damping and two-magnon scattering. Phys., Rev. B 76, 104416 (2007).

32. Mizukami, S. et al. The study on ferromagnetic resonance linewidth for $\mathrm{NM} / 80 \mathrm{NiFe} / \mathrm{NM}(\mathrm{NM}=\mathrm{Cu}, \mathrm{Ta}, \mathrm{Pd}$ and Pt) films. Jpn. J. Appl. Phys. 40, 580-585 (2001)

33. Skrotskii, G. V. \& Kurbatov, L. V. Theory of the anisotropy of the width of ferromagnetic resonance absorption line. Sov. Phys. JETP 35 148-151 (1959).

34. Suhl, H. Ferromagnetic resonance in Nickel ferrite between one and two kilomegacycles. Phys. Rev. 97, 555-557 (1955).

35. Tserkovnyak, Y. et al. Spin pumping and magnetization dynamics in metallic multilayers. Phys. Rev. B 66, 224403 (2002)

36. Pai, C. F. et al. Dependence of efficiency of spin Hall torque on the transparency of Pt/ferromagnetic layer interfaces. Phys. Rev. B 92, 064426 (2015)

37. Wastlbauer, G. \& Bland, J. A. C. Structural and magnetic properties of ultrathin epitaxial Fe films on GaAs (001) and related semiconductor substrates. Adv. Phys. 54, 137-219 (2005).

38. Gordon, R. A. \& Crozier, E. D. In-plane structure anisotropy of ultra-thin Fe films on GaAs (001)- 4×6: X-ray absorption fine structure spectroscopy measurements. Phys. Rev. B 74, 165405 (2006).

39. Moser, J. et al. Tunneling anisotropic magnetoresistance and spin-orbit coupling in Fe/GaAs/Au tunnel junctions. Phys. Rev. Lett. 99, 056601 (2007)

40. Ebert, H. et al. Calculating condensed matter properties using the KKRGreen's function method-recent developments and applications. Rep. Prog. Phys. 74, 096501 (2011).

\section{Acknowledgements}

The authors thank M. Gmitra and J. Fabian for fruitful discussions. L. Chen is grateful for support from the Alexander von Humboldt Foundation. This work is support by the German Science Foundation (DFG) via SFB 689 and SFB 1277.

\section{Author contributions}

L.C., C.H.B. and D.W. planned the study. L.C. fabricated the devices, and collected and analysed the data. M.A.W.S. carried out the full-film FMR measurements and L.C. analysed the data. H.S.K. performed the magnetization measurements. M.K., D.S. and D.B. grew the samples. S.W., S.M. and H.E. carried out the first-principle calculations. L.C. wrote the manuscript with input from S.W., S.M., H.E., C.H.B. and D.W. All authors discussed the results.

\section{Competing interests}

The authors declare no competing interests.

\section{Additional information}

Supplementary information accompanies this paper at https://doi.org/10.1038/s41567018-0053-8.

Reprints and permissions information is available at www.nature.com/reprints. Correspondence and requests for materials should be addressed to C.H.B. Publisher's note: Springer Nature remains neutral with regard to jurisdictional claims in published maps and institutional affiliations. 


\section{Methods}

Sample preparation. $\mathrm{Fe} / \mathrm{GaAs}$ samples are grown by molecular beam epitaxy (MBE) in a MBE cluster without being exposed to air to guarantee a high-quality interface. First, an undoped GaAs buffer layer $(100 \mathrm{~nm})$ is deposited on top of a GaAs (001) semi-insulating substrate at $560^{\circ} \mathrm{C}$ in the III-V MBE chamber.

A clear $(2 \times 4)$ surface reconstruction pattern has been observed, indicating an As-terminated flat surface. Then, without breaking the vacuum, the wafer is transferred to a metal MBE chamber, where Fe films with different set thickness $t$ are deposited on the same wafer at a substrate temperature of $75^{\circ} \mathrm{C}$ by controlling the main shutter. Two series of samples have been prepared: $\mathrm{S} 1(t=5,3.9,1.9$, $1.3 \mathrm{~nm})$ and $\mathrm{S} 2(t=1.1,0.9 \mathrm{~nm})$, here the set thickness is the nominal thickness determined by a quartz thickness monitor. Note that, in the ultrathin limit, it is hard to determine the thickness accurately. However, within one growth series, the relative thickness can be given. The absolute value differs between different sample series. A streaky reflection high-energy electron diffraction pattern and reflection high-energy electron diffraction oscillations have been observed for all of the samples, indicating the epitaxial growth mode as well as the flat interface between $\mathrm{Fe}$ and GaAs. To avoid Fe oxidation, finally, a 3-nm-thick Al capping layer is deposited onto the Fe film.

Device. For the SO-FMR measurements, the stripe dimension is $6.4 \mu \mathrm{m} \times 100.0 \mu \mathrm{m}$ $(20 \mu \mathrm{m} \times 100.0 \mu \mathrm{m})$ for sample series S1 (S2), which are defined by electron-beam lithography and ion-beam etching. All of the stripes are along the [100] orientation. The contacts are made from $15 \mathrm{~nm}$ Ti and $150 \mathrm{~nm} \mathrm{Au}$.

Measurements. For the spin-orbit ferromagnetic resonance measurement, a bias tee is used to separate the d.c. voltage from the microwave background. The microwave input power is $22 \mathrm{dBm}$. For the magnetic-field-angle-dependent measurement, the microwave frequency was $12 \mathrm{GHz}$ for S1 and $13 \mathrm{GHz}$ for S2. All measurements have been performed at room temperature.

Data availability. The data that support the plots within this paper and other findings of this study are available from the corresponding author upon reasonable request. 